\title{
A stable genetic polymorphism underpinning microbial syntrophy
}

\author{
Tobias Großkopf ${ }^{1}$, Simone Zenobi ${ }^{1}$, Mark Alston ${ }^{2}$, Leighton Folkes ${ }^{2}$, David Swarbreck ${ }^{2}$ \\ and Orkun S Soyer ${ }^{1}$ \\ ${ }^{1}$ School of Life Sciences, The University of Warwick, Coventry, UK and ${ }^{2}$ The Genome Analysis Centre, \\ Norwich Research Park, Norwich, UK
}

\begin{abstract}
Syntrophies are metabolic cooperations, whereby two organisms co-metabolize a substrate in an interdependent manner. Many of the observed natural syntrophic interactions are mandatory in the absence of strong electron acceptors, such that one species in the syntrophy has to assume the role of electron sink for the other. While this presents an ecological setting for syntrophy to be beneficial, the potential genetic drivers of syntrophy remain unknown to date. Here, we show that the syntrophic sulfate-reducing species Desulfovibrio vulgaris displays a stable genetic polymorphism, where only a specific genotype is able to engage in syntrophy with the hydrogenotrophic methanogen Methanococcus maripaludis. This 'syntrophic' genotype is characterized by two genetic alterations, one of which is an in-frame deletion in the gene encoding for the ion-translocating subunit cooK of the membrane-bound COO hydrogenase. We show that this genotype presents a specific physiology, in which reshaping of energy conservation in the lactate oxidation pathway enables it to produce sufficient intermediate hydrogen for sustained $M$. maripaludis growth and thus, syntrophy. To our knowledge, these findings provide for the first time a genetic basis for syntrophy in nature and bring us closer to the rational engineering of syntrophy in synthetic microbial communities.
\end{abstract}

The ISME Journal (2016) 10, 2844-2853; doi:10.1038/ismej.2016.80; published online 3 June 2016

\section{Introduction}

Syntrophic interactions represent cases of metabolic cooperation between two phenotypically distinct organisms (Schink, 1997; McInerney et al., 2008; Sieber et al., 2012; Morris et al., 2013). These interactions are common among microbes living in environments that can be readily depleted of strong electron acceptors. In such environments, including anaerobic reactors, animal guts, ocean and lake sediments and soil, the depletion of suitable electron acceptors is expected to increase the abundance of microbes with fermentative metabolism. The low thermodynamic energy associated with fermentative metabolic pathways results in 'thermodynamic inhibition' of microbial growth due to product accumulation (Schink, 1997; Kleerebezem and Stams, 2000; Großkopf and Soyer, 2014, 2016). This inhibition of growth in the primary degrading microbes can be lifted by others consuming the inhibitory waste product (mostly hydrogen) (Seitz et al., 1988; Schink, 1997; Sieber et al., 2012). Thus, environmental depletion of electron acceptors provides a

Correspondence: T Großkopf or Orkun S Soyer, School of Life Sciences, The University of Warwick, Coventry CV4 7AL, UK. E-mail: t.grosskopf@warwick.ac.uk or O.Soyer@warwick.ac.uk Received 3 January 2016; revised 28 March 2016; accepted 1 April 2016; published online 3 June 2016 setting in which syntrophic interactions can more readily emerge. Besides their central role in natural microbial communities (McInerney et al., 2009; Sieber et al., 2012), syntrophic interactions also constitute a desired motif in engineered synthetic microbial communities, where they can provide a metabolic basis for stable community formation (Gilbert et al., 2003; Großkopf and Soyer, 2014; Santala et al., 2014).

Despite their importance in ecological and engineered settings, our understanding of the molecular basis of syntrophic interactions remains limited. Transcriptomic analyses in a syntrophic model system (Walker et al., 2012, 2009) have highlighted several genes that are differentially regulated under conditions of syntrophy. The gene products are involved in primary energy metabolism as well as in secretion of secondary metabolites which are suggested to enhance the growth of the syntrophic partner (Walker et al., 2009, 2012). Other studies indicated genes involved in formation of spatial structures to be important for syntophic interactions (Schink, 1997; Kato and Watanabe, 2010; Summers et al., 2010). Indeed, biofilm or granule formation (Summers et al., 2010; Wintermute and Silver, 2010a; Worm et al., 2014), as well as direct attachment via flagella (Marx, 2009; Shimoyama et al., 2009; Kato and Watanabe, 2010), is observed in several co-cultures involving syntrophy or cross- 
feeding. However, formation of spatial structures like biofilms is also frequently observed in monocultures (Zhang et al., 2007) and under non-syntrophic conditions (Nadell et al., 2009), and as such, it is not clear whether biofilm formation is a key driver in the formation and maintenance of syntrophies.

At a cellular level, it is well documented that syntrophic associations result in transcriptomic and metabolomic changes in the involved organisms (Walker et al., 2009, 2012; Qi et al., 2014). The study of these changes has led to the identification of specific genes, whose deletion completely abolishes the ability to form syntrophic interactions (Walker et al., 2009). Despite these important insights, it is still not clear whether there are specific genetic alterations that can enhance formation of syntrophies. If such alterations exist, then identifying these could allow for a better understanding of the ecological and evolutionary basis of natural syntrophies and enable engineering of synthetic ones. The latter objective is motivated by observations that synthetic engineering of syntrophic and mutualistic interactions among microbes can increase bioproductivity (Shou et al., 2007; Wintermute and Silver, 2010b; Kerner et al., 2012; Mee et al., 2014) and scope of biotechnological applications (Gilbert et al., 2003; Bernstein et al., 2012; Santala et al., 2014).

Here, we aim to identify genetic drivers of syntrophy by focusing on physiological and genetic analysis of the well-established model syntrophic system between a sulfate-reducing bacteria (SRB) Desulfovibrio vulgaris strain Hildenborough (DvH) and a hydrogenotrophic methanogen Methanococcus maripaludis S2 (Mm) (Stolyar et al., 2007; Hillesland and Stahl, 2010). A typical SRB, DvH is equipped with a dissimilatory sulfate reductase and various hydrogenases (Pieulle et al., 2005), which allows it to utilize hydrogen, while reducing sulfate. The ability to utilize hydrogen makes SRB a competitor for methanogens like $\mathrm{Mm}$ in the presence of sulfate. In the absence of sulfate, however, its fermentative metabolism results in the production of hydrogen and opens up the potential of a syntrophic interaction with $\mathrm{Mm}$.

We show that this ability to engage in syntrophy with $\mathrm{Mm}$ is underpinned by a stable genetic polymorphism in $\mathrm{DvH}$; under a sulfate-free, minimal lactate media, only a specific genotype of $\mathrm{DvH}$ can grow syntrophically with $\mathrm{Mm}$. We find that this genotype naturally exists and is characterized by a set of two distinguishing genetic mutations. Both mutations are linked to the lactate oxidation pathway, the energetics of which is a key driver of the syntrophic interaction. In particular, the resulting altered thermodynamics of this pathway increases the tolerated hydrogen pressure of lactate oxidation in $\mathrm{DvH}$, therefore providing substrate for growth of $\mathrm{Mm}$ and a stable syntrophy can be formed. These findings provide the first evidence of a specific syntrophy-inducing genetic polymorphism in SRB and point toward energetic determinants of metabolism as a key driver for syntrophy formation in general.

\section{Materials and methods}

\section{Culturing and media}

Cultures of Desulfovibrio vulgaris strain Hildenborough (DSM644, DvH) and Methanococcus maripaludis S2 (DSM2067, Mm) were ordered from DSMZ (www.dsmz.de). Cultures of $\mathrm{Mm}$ were grown in medium 141, as specified on the DSMZ homepage, or in Co-Culture Medium (CCM) (Walker et al., 2009) with slight modifications (for details, see Supplementary Methods). Cultures of DvH were grown in Postgate medium C (Postgate, 1984) (for DNA extraction and plating) or in CCM. Co-cultures of $\mathrm{DvH}$ and $\mathrm{Mm}$ were grown in CCM. Initial cocultures were started by injecting $1 / 20$ volume of each CCM-washed monoculture into fresh CCM. Culturing conditions were $37^{\circ} \mathrm{C}$ in a static incubator (Heratherm, Thermo Scientific, Waltham, MA, USA) in the dark unless otherwise specified. The culture vessels used were either anaerobe tubes (Chemglass Life Sciences, Vineland, NJ, USA) or 50 and $100 \mathrm{ml}$ serum flasks (Supelco, Bellefonte, PA, USA) with blue butyl rubber stoppers (Chemglass Life Sciences). Subculturing was performed by injecting $1 / 20$ of the volume of a late log-phase culture into fresh culture medium (for example, $250 \mu \mathrm{l}$ into $5 \mathrm{ml}$ ). The detailed recipes and preparation protocols of the media used are supplied in the Supplementary Methods.

\section{Optical density and gas production}

Optical density (OD) was measured at $600 \mathrm{~nm}$ in a spectrophotometer (Thermo Scientific). Gas measurements were performed on an Agilent $7890 \mathrm{~A}$ Gas chromatograph (Agilent Technologies, Craven Arms, UK). For detailed procedures and calculations for both OD and gas measurements, see Supplementary Methods.

\section{Microscopy}

For microscopic analysis, $5 \mu \mathrm{l}$ of culture was placed on a microscope glass slide and covered with a glass coverslip. The coverslip was sealed to the slide with transparent nail-varnish to minimize evaporation. Slides were placed on an inverted fluorescence microscope (IX83, Olympus, Tokyo, Japan) and observed under $\times 600$ magnification in phase contrast or using a blue light excitation at $440 \mathrm{~nm}$ generated by a pE-2 LED system (CoolLed, Andover, MA, USA) and a band-pass CFP filter at $503-538 \mathrm{~nm}$ (Olympus). Mm has a co-factor $\left(\mathrm{F}_{420}\right)$ that shows blue-green fluorescence upon illumination with blue light (Peak $E x / E m=420 / 470 \mathrm{~nm}$ ) (Doddema and Vogels, 1978). Images were recorded in black and white on a CoolSnap $\mathrm{HQ}^{2}$ camera (Photometrics, Tucson, AZ, USA) and false (blue) 
color added to the fluorescent channel image. Image overlays were generated in the camera software cellSens Dimensions (Olympus) and exported as image files.

\section{DNA extraction}

For DNA extraction, $30 \mathrm{ml}$ of DvH cultures was grown in liquid culture (Medium C) until late logphase. Cells were harvested by centrifugation at $12300 \mathrm{~g}$ for $3 \mathrm{~min}$ in a tabletop centrifuge (Microfuge SCF2, Stuart, Stone, UK) and the resulting pellet frozen at $-20^{\circ} \mathrm{C}$ until DNA extraction using the Wizard gDNA purification kit for Gramnegative bacteria (Promega, Fitchburg, WI, USA). Extraction resulted in 110-200 $\mu \mathrm{g}$ of DNA per sample. This still was below the purity requirements for Illumina sequencing as confirmed by $260 / 280 \mathrm{~nm}$ and $260 / 230 \mathrm{~nm}$ absorbtion ratio measurements $(\sim 1.8$ and $\sim 1.4$, respectively) on a CLARIOstar plate reader (BMG Labtech, Ortenberg, Germany) equipped with LVis plate (BMG Labtech). For further purification, extracted DNA was bound to a purification column of the PureLink Genomic DNA Mini Kit (Invitrogen, Carlsbad, CA, USA) and eluded in buffer. In detail, $40 \mu$ of extracted gDNA samples was treated with the following steps: add $200 \mu \mathrm{l}$ milliq $-\mathrm{H}_{2} \mathrm{O}$, add $200 \mu \mathrm{l}$ PureLink GenomicLysis/Binding Buffer, vortex at 2200 r.p.m. for 5 s, add $200 \mu$ l ethanol (100\%), vortex

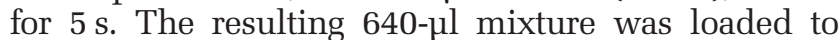
a PureLink Spin Column and from there on, the manufacturer's instructions for DNA purification were followed. The resulting DNA quantity was about 1/6 of the original sample ( $10 \mu \mathrm{g}$ per sample); however, the $260 / 280 \mathrm{~nm}$ and $260 / 230 \mathrm{~nm}$ ratios were around 1.8 and 2.2, respectively, which indicates high purity DNA samples.

\section{Library preparation and MiSeq sequencing}

Illumina TruSeq DNA libraries were prepared at The Genome Analysis Centre (TGAC, Norwich, UK) following the manufacturer's protocol (15036187; Illumina, San Diego, CA, USA) with some minor modifications. In brief, samples from DvH-s and DvH-ns clones were normalized to $1 \mu \mathrm{g}$ of input DNA and sheared by sonication via a Covaris S2 to $\sim 500$ bp long fragments. Following ligation of indexing adapters to the DNA fragments, samples were amplified by PCR. The insert size of the DNA libraries was verified on a PerkinElmer GX using the High Sensitivity DNA chip and the concentration determined by a High Sensitivity Qubit assay. Beckman Coulter XP beads (Part No: A63880) were used for size selection. The resulting 10 genomic DNA paired-end libraries were pooled, spiked with 1\% PhiX Control v3 and sequenced with $150 \mathrm{bp}$ pairedend run metrics on the Illumina MiSeq platform (MiSeq Reagent Kit v2, using MiSeq Control Software 2.5.0 and RTA 1.18.54). The fastq data files were deposited in the European Nucleotide Archive and may be accessed at http://www.ebi.ac.uk/ena/ data/view/PRJEB12162.

\section{Variant calling}

The reads from each sample were quality trimmed using Sickle (Joshi and Fass, 2011) and mapped independently to the DvH reference genome (http:// bacteria.ensembl.org/desulfovibrio_vulgaris_str_ hildenborough/Info/Index/, accessed 9/2/15) using the sequence aligner BWA-SW v0.7.12 (Li and Durbin, 2010). The resulting alignments were converted into binary format and position sorted using SAMtools v1.1 (Li et al., 2009). To call singlenucleotide polymorphisms (SNPs) and insertions/ deletions (INDELS), SAMtools' mpileup was used on the processed alignments and the output passed onto VarScan v2.3.7 which was run with default settings (Koboldt et al., 2012). The effects of the called variants within the resulting VCF files produced by VarScan were annotated using SnpEff v4.0 (Cingolani et al., 2012).

\section{DvH core metabolic pathways and their thermodynamic representation}

A representation of the core metabolic (catabolic) system of DvH (as shown in Figure 3) was produced based on information from the literature (Stolyar et al., 2007; Walker et al., 2009) and bioinformatics databases Rhea (http://www.rhea-db.org/home) (Morgat et al., 2015), ChEBI (https://www.ebi.ac.uk/chebi/) (Hastings et al., 2013) and IMG (https://img.jgi.doe.gov/cgi-bin/ $\mathrm{m} / \mathrm{main}$.cgi) (Markowitz et al., 2012). The full chemical formula for each reaction, as well as their thermodynamic data, is given in Supplementary Table S1, while chemical abbrevations used are further described in Figure 3 legend and Supplementary Table S2. The compiled thermodynamic values listed in Supplementary Table S1 are taken from the literature (Thauer et al., 1977) and used to compute redox potentials for electron accepting or donating reactions as shown in Figure 3. The downward shift in redox potential of Coo in Figure 3 is calculated based on a transport of two single charged ions per hydrogen produced over a 100-fold concentration gradient across the membrane $\left(\sim-23 \mathrm{~kJ} \mathrm{~mol}^{-1}\right)$.

\section{Results and discussion}

DvH-Mm syntrophy requires a specific DvH genotype To study the potential genetic basis of syntrophic interactions, we used here the model system of $\mathrm{DvH}$ and $\mathrm{Mm}$. In the absence of sulfate, and under minimal, lactate-containing media, the DvH-Mm co-culture has been shown to syntrophically oxidize lactate to produce methane, acetate and $\mathrm{CO}_{2}$ while relying mainly on hydrogen exchange between the two species (Stolyar et al., 2007). When we attempted to initialize this system, the initial frequency of co-culture success, assessed with 
methane formation and OD measurements, was only 1/40 (Supplementary Figure S1a). This observation can be explained by the presence of a low frequency phenotypic or genetic variant in the wild-type population of the $\mathrm{DvH}$ that is characterized by its ability to form a successful syntrophy with Mm. In the case of phenotypic variability, where a single genotypic background gives rise to multiple phenotypes (Balaban et al., 2004), it would be expected that DvH clones isolated from the working syntrophic coculture and re-cultured with $\mathrm{Mm}$ should again lead to low frequency in co-culture success. In contrast, in the case of genotypic variability, we would expect DvH clones isolated from the working syntrophic coculture to give much higher co-culture success when re-cultured with the $\mathrm{Mm}$.

To test these possibilities, we isolated 20 clones of DvH both from the working syntrophic co-culture and from the original inoculum culture of $\mathrm{DvH}$. Re-inoculating these $40 \mathrm{DvH}$ clones with $\mathrm{Mm}$ (see Materials and methods, Supplementary Figure S1b), we found that all co-cultures involving clones isolated from the working syntrophic co-culture led to increased OD and methane production after 1 week of growth (Figure 1). For the 20 co-cultures started with clones from the working syntrophic co-culture, the average methane production after 1 week was $1.13 \mathrm{ml}( \pm 0.64 \mathrm{ml}, n=20)$, while it was $0.18 \mathrm{ml}( \pm 0.03 \mathrm{ml}, n=20)$ for the co-cultures started with clones from the original $\mathrm{DvH}$ population. The pattern was consistent after subculturing (1/20 inoculum), with the second generation of cultures producing $1.03 \mathrm{ml}( \pm 0.46 \mathrm{ml}, n=20)$ and $0.07 \mathrm{ml}$ $( \pm 0.05 \mathrm{ml}, n=20)$ methane for $\mathrm{DvH}$ clones isolated from the working syntophic co-culture and the

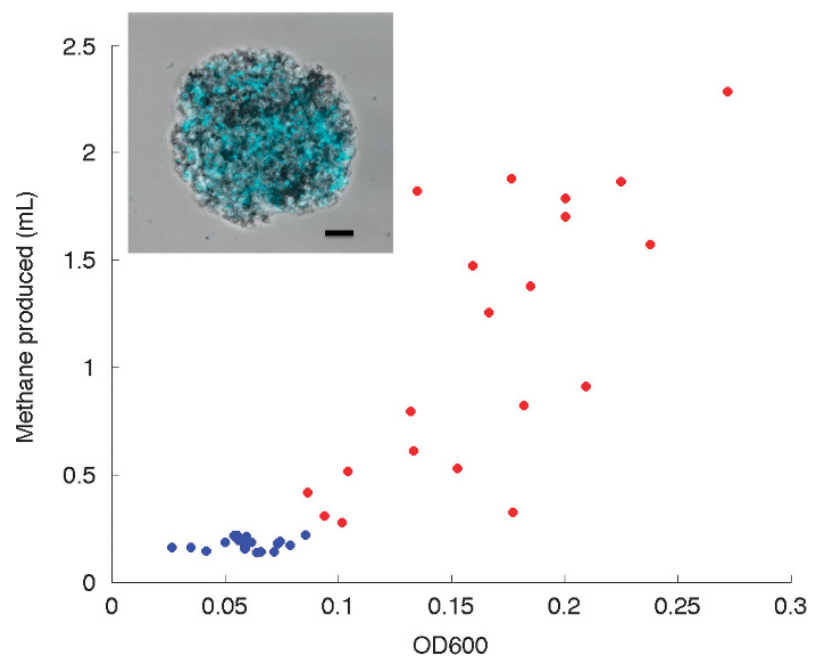

Figure 1 OD at $600 \mathrm{~nm}$ (OD600) and methane production of DvHns/Mm (blue, 20 replicates) and DvH-s/Mm co-cultures (red, 20 replicates) grown on CCM (see Materials and methods). The inset shows a sample, phase-contrast image of a granule from a DvH-s/ $\mathrm{Mm}$ co-culture taken at $\times 600$ magnification, and overlayed with the emission from the blue fluorescence channel. Scale bar $=10 \mu \mathrm{m}$. The blue autofluorescence originates from the $\mathrm{F}_{420}$ cofactor of the methanogen. original culture, respectively. For the successful co-cultures, we also observed a positive correlation between the methane production and OD $\left(r^{2}=0.54\right.$, $\left.\mathrm{V}(\mathrm{ml})_{\mathrm{CH} 4}=5.23 \times \mathrm{OD}-0.44\right)$; however, the latter is not a good predictor of growth due to a high degree of granule formation in the co-culture and associated patchiness (see Figure 1, inset). The consistent co-culturing success of $\mathrm{DvH}$ clones isolated from the working syntrophic co-culture leads to the conclusion that the observed success in formation of syntrophy is due to a genetic factor and not due to phenotypic variability. Hereafter, we refer to the clone leading to syntrophic co-cultures with $\mathrm{Mm}$ as 'DvH-s' and to the other clone as 'DvH-ns', for 'nonsyntrophic'.

\section{Genetic analysis of DvH-s vs DvH-ns reveals} determinants of syntrophy

To better understand the genetic difference between these two genotypes, we isolated five new clones of $\mathrm{DvH}$-s from a successful co-culture and five clones from the original $\mathrm{DvH}$ population, which were expected to be DvH-ns. We re-sequenced the genomes of all of these 10 clones and annotated each genome against the reference $\mathrm{DvH}$ genome, which contains the main chromosome and a megaplasmid (Heidelberg et al., 2004) (see Materials and methods). We found a total of 57 polymorphisms across the 10 clones. There were a total of 26 SNPs on the chromosome (Figure 2) and 2 on the megaplasmid, as well as 27 INDELs on the chromosome (Figure 2) and 2 more on the megaplasmid. A detailed view of the genotype of each strain for all 57 positions is provided in Supplementary File 1.

To verify again the ability of each of these sequenced clones to form a successful syntrophy or not, we co-cultured them with $\mathrm{Mm}$. These experiments revealed that as expected all five DvH-s clones (labeled as DvH-s 1-5) were able to generate a methane producing, syntrophic co-culture with $\mathrm{Mm}$ (Figure 3). Among the DvH-ns clones, three resulted in no co-culture growth as expected, while two (initially labeled as DvH-ns 1 and DvH-ns2) did result in relatively successful syntrophic co-culture (Figure 3) (these findings are further discussed below). Taking these physiological results in accord, we analyzed the sequence data for genetic changes that are common to all seven clones that led to successful co-cultures, and that are lacking from the other three clones. Out of all 57 mutations, only 2 were present in all 7 'co-culturable' clones, but absent in clones DvH-ns 3-5 (Figure 2), suggesting that these two mutations are essential for syntrophy. The first of these two mutations is a nonsynonymous point mutation from $\mathrm{G}$ to $\mathrm{A}$ in the gene DVU3023 (DvH chromosome location: 3142197), resulting in an amino-acid exchange from Aspartate to Asparagine. The DVU3023 gene is annotated to encode for a sigma-54-dependent response regulator that controls the downstream operon (DVU3025- 


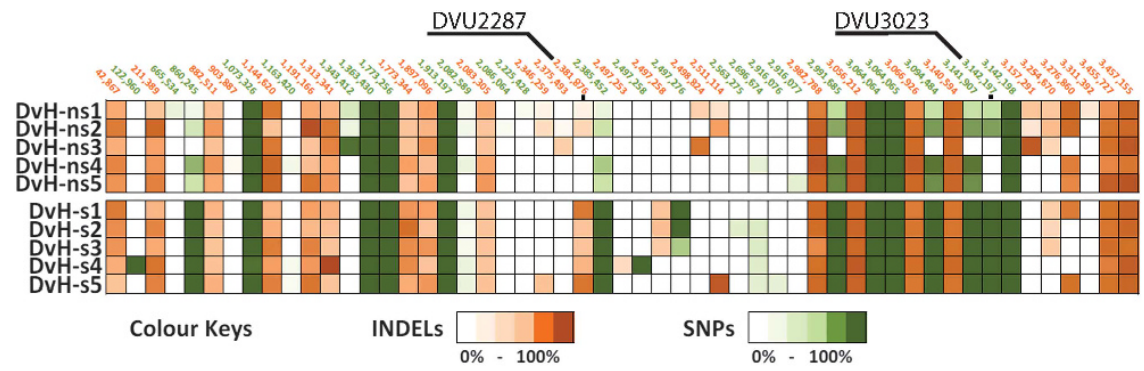

Figure 2 Mutations identified on the genome of 10 clones isolated from a working syntrophic co-culture (clones initially labeled as DvH-s 1-5) and from the wild-type DvH population (clones initially labeled as DvH-ns 1-5). Each square represents an SNP (green) or INDEL (brown), as identified by comparing the genome sequence of the corresponding clone with the reference DvH genome. The color scale of each square encodes the proportion of reads that contain the corresponding SNP or INDEL. The genomic location of each mutation is shown at the top. In total, 26 SNPs and 27 INDELs were identified on the chromosome. An additional two SNPs and two INDELs were identified on the megaplasmid that are not shown in this figure (but included in the Supplementary File 1). As discussed in the main text, 2 out of the 57 mutations were present in all seven 'co-culturable' clones (DvH-s 1-5, DvH-ns 1 and DvH-ns2), a synonymous point mutation in the gene DVU3023 and a disruptive INDEL in the gene DVU2287.

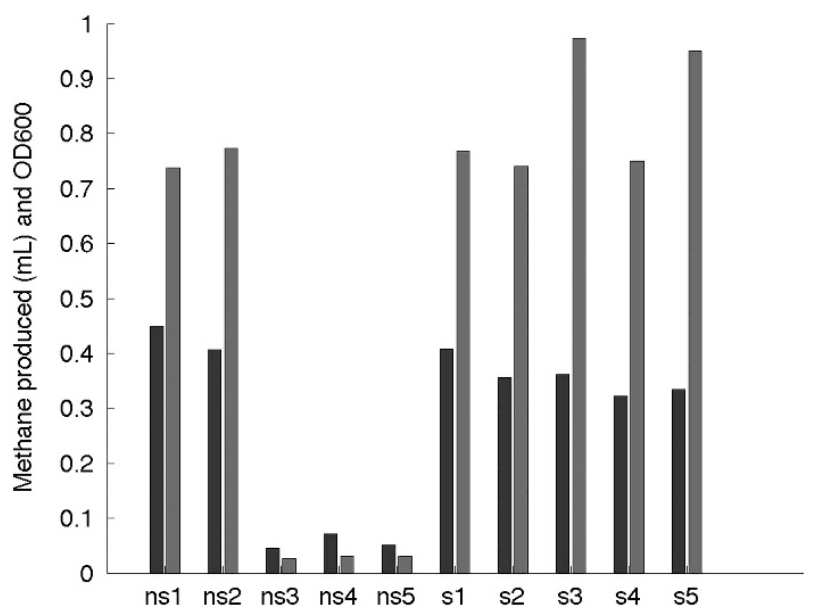

Figure 3 OD600 (blue) and methane production (red) of five DvH-ns and DvH-s clones respectively, when grown in co-culture with Mm in CCM (see Materials and methods). A full color version of this figure is available at the The ISMEJ Journal journal online.

DVU3033), which includes the genes lactate permease and pyruvate decarboxylase. Notably, both enzymes are involved in the lactate oxidation pathway, with the first enzyme catalyzing the lactate uptake and the second catalyzing conversion of the pyruvate resulting from lactate oxidation. The second mutation is an in-frame deletion that led to the removal of a GAG sequence in the gene DVU2287 (DvH chromosome location: 2381876), resulting in the replacement of a TGA codon with a TCG codon. DVU2287 is annotated to encode for the CooK subunit of Coo, which is annotated as the $\mathrm{H}^{+} / \mathrm{Na}^{+}$ ion-translocating subunit of the carbon monooxidedependent, membrane-bound dehydrogenase, which is hypothesized to be involved in the lactate oxidation pathway (Walker et al., 2009). The TGA codon is known to encode for a stop codon, which can either lead to a truncated gene product or introduction of a Selenocysteine residue during translation (Valente et al., 2005). Thus, in all seven genotypes able to form syntrophy, this replacement of the TGA codon with the TCG codon either resulted in a gain-of-function at the DVU2287 gene or to an exchange of a Selenocysteine residue with a Serine residue. Interestingly, sequencing data revealed that clones DvH-ns 1 and DvH-ns2, which were picked from the original $\mathrm{DvH}$ population but could form syntrophy with $\mathrm{Mm}$, show a mixed signature of alleles consisting of the mutated and non-mutated sequences at this locus (Figure 2). This could be explained by the initial clone picking resulting in a heterogeneous sample of genotypes, or by the presence of multiple genome copies within one cell-a case that is known to be common in SRB (Postgate et al., 1984).

\section{A thermodynamic model highlights potential implications of the cooK mutation}

Given the well-established role of thermodynamic limitations as the basis of syntrophic interactions and the role of hydrogen formation in this (CordRuwisch et al., 1988; Schink, 1997; Noguera et al., 1998), it is possible that the identified mutation in the Coo hydrogenase relates to the thermodynamics of the lactate oxidation pathway. To explore this possibility, we performed a thermodynamic analysis of this pathway as it is seen in $\mathrm{DvH}$ (see Materials and methods and Supplementary Table S1). As expected, this thermodynamic view shows the feasibility of lactate oxidation in the presence of sulfate, where electrons can flow from the lactate dehydrogenase (LDH) catalyzed reaction to sulfate reduction reactions, in accordance with the standard reduction potentials of these reactions (Figure 4). This would allow $\mathrm{DvH}$ to harvest the available energy for growth. In the absence of sulfate as a terminal electron acceptor, however, lactate oxidation would only be possible if the LDHcatalyzed reaction could be coupled with an appropriate electron accepting reaction. Suitable reactions for this role, and available in the central metabolism 
of $\mathrm{DvH}$, would include the reduction of acetaldehyde to ethanol and reduction of pyruvate to alanine. Combining the lactate oxidation with either of these
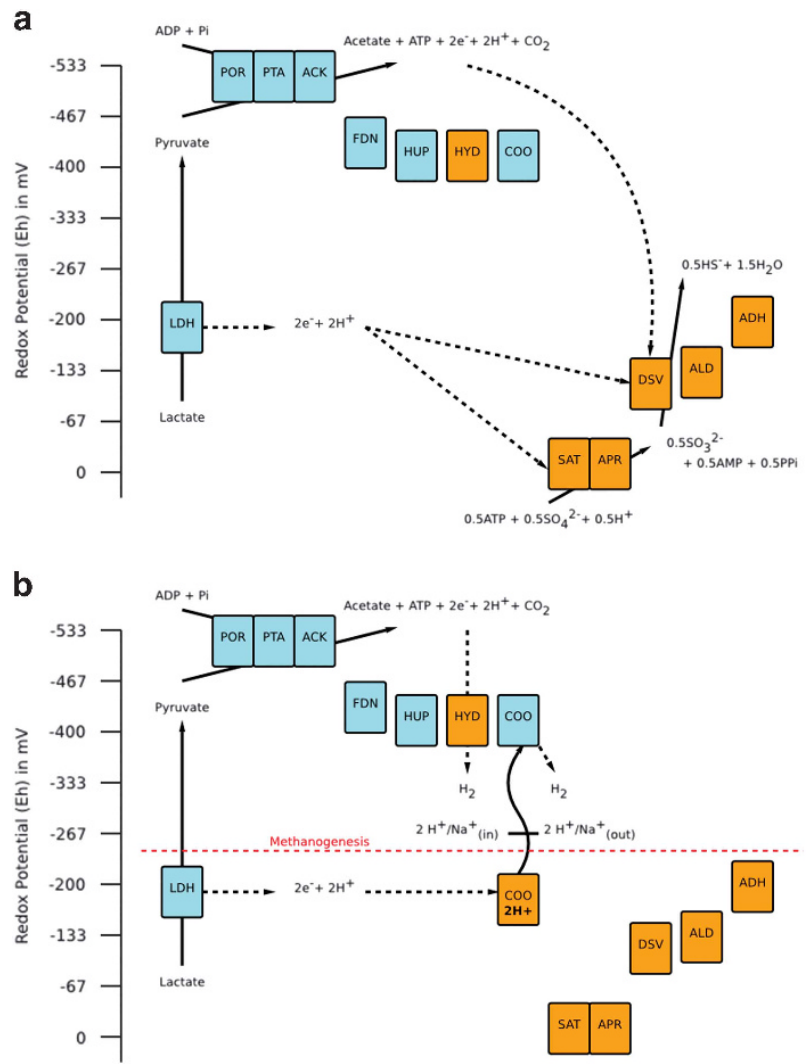

Figure 4 A cartoon representation of the lactate oxidation and sulfate respiration pathways in DvH. Each of the enzymes involved in these pathways is placed on the cartoon according to the standard reduction potentials of the redox reactions that they catalyze (see Supplementary Table S1). In the lactate pathway, lactate is first oxidized to pyruvate and then to acetate, each reaction releasing two electrons and two protons. The protons can then be reduced to produce hydrogen, in a reaction catalyzed by the different hydrogenases. The enzymes catalyzing electron releasing and accepting reactions are shown in blue and orange, respectively (enzymes that are connected represent combined reactions). The solid and dashed arrows represent the flow of matter and electrons, respectively. Note that due to thermodynamics, electrons can only flow from reactions with more negative reduction potentials to reactions with more positive reduction potentials. (a) In the presence of sulfate, lactate oxidation can be coupled to the electron accepting reactions of the sulfate pathway. (b) In the absence of sulfate, it is predicted that electrons from lactate oxidation flow to proton reduction mediated by Coo. The reduction potential of that reaction is predicted to be shifted downwards by energy investments from translocating two sodium/proton across a membrane gradient. The added energy allows an equilibrium hydrogen pressure equal to the other hydrogenases, acting as an elevator for electrons (the curved arrow). The horizontal dashed red line is the redox potential at which hydrogentrophic methanogens are assumed to accept electrons $(-244 \mathrm{mV})$, and is included as a reference point. The enzyme labels are $\mathrm{LDH}=$ lactate dehydrogenase, $\mathrm{POR}=$ pyruvate-ferredoxin $\quad$ oxidoreductase,$\quad \mathrm{PTA}=$ phosphate acetyltransferase, ACK = acetate kinase, $\mathrm{FDN}=$ formate dehydrogenase, HUP = uptake hydrogenase, $\mathrm{HYD}=$ release hydrogenase, $\mathrm{COO}=$ Coo hydrogenase (potentially $\mathrm{H}^{+} / \mathrm{Na}^{+}$dislocating), $\mathrm{SAT}=$ ATP-sulfate adenylyltransferase, APR =adenylyl sulfate reductase, $\quad \mathrm{DSV}=$ sulfite reductase, $\mathrm{ALD}=$ alanine dehydrogenase, $\mathrm{ADH}=$ ethanol dehydrogenase. reactions would lead to a thermodynamically feasible pathway; however, they would result in the bypassing of the ATP generating step of the lactate oxidation pathway; the conversion of pyruvate to acetate mediated by the enzymes pyruvate oxidoreductase, phosphate acetyltransferase and acetate kinase (POR-PTA-ACK in Figure 4). A third option as an electron accepting reaction would be the hydrogenase-catalyzed proton reduction, leading to hydrogen formation (Walker et al., 2009) (Figure 4). Coupling this reaction with lactate oxidation would allow production of pyruvate, with subsequent pyruvate oxidation via POR-PTA-ACK pathway leading to ATP formation.

The free energy of the combined lactate oxidationproton reduction reaction under standard conditions is, however, strongly positive (43.2 $\left.\mathrm{kJ} \mathrm{mol}^{-1}\right)$, creating an uphill energy barrier for growth (see Materials and methods, Supplementary Table S1). Changing this energy budget in order to make this fermentative reaction thermodynamically feasible would require reducing the hydrogen and pyruvate concentrations. In particular, assuming biologically realistic concentrations (Bennett et al., 2009) of $1 \mathrm{mmol}$ and $0.1 \mathrm{mmol}$ for lactate and pyruvate respectively, then the hydrogen partial pressure (that is, concentration) required to achieve a negative reaction free energy would be around 0.03Pa. This pressure is well below the thermodynamically allowed minimum hydrogen pressure of $0.2 \mathrm{~Pa}$ required for hydrogenotrophic methanogenesis (Schink, 1997). Thus, at the possible hydrogen pressures that can lead to syntrophy, the fermentative lactate oxidation metabolism might stall due to thermodynamic limitations. One way to overcome this thermodynamic limitation would be to invest additional energy into the lactate oxidation reaction, with the side effect that a higher hydrogen pressure would result under physiological conditions. As the Coo is annotated as an $\mathrm{H}^{+} / \mathrm{Na}^{+}$ion-translocating hydrogenase, we hypothesize that the identified polymorphism in this gene increases the number of ions it can translocate over the membrane per number of hydrogen produced, and thereby use the membrane gradient as a form of cellular energy to invest in lactate oxidation. In particular, each ion translocated per hydrogen produced could result up to a hundred-fold increase in equilibrium hydrogen pressure tolerated by $\mathrm{DvH}$ (that is, from $0.03 \mathrm{~Pa}$ to $3 \mathrm{~Pa}$ for single ion, assuming $2 \mathrm{pH}$ units membrane gradient). In a view based on electron flow, this scenario could be seen as shifting the reduction potential of the Coo-catalyzed proton reduction reaction to more positive values, and thereby making it thermodynamically feasible for this reaction to accept electrons from $\mathrm{LDH}$-mediated lactate oxidation reaction (Figure 4). We also note that the function of hydrogenases, and in particular their ability to shuttle hydrogen and protons to the reaction center, commonly involves selenocysteine and cysteine residues (Valente et al., 2005; Arnér, 2010). As such, it is tempting to speculate that the mutation observed here in the cooK gene involves the replacement of a 
selenocysteine residue with serine in the cooK gene product, with direct effect on enzyme kinetics or reduction potential of the catalyzed proton reduction, as seen in some hydrogenases (Arnér, 2010; GutiérrezSánchez et al., 2010). A sequence analysis shows that many SRBs contain a homolog of the CooK subunit and carry a conserved cysteine residue in the identified loci (Supplementary File 2).

Physiology of DvH-s is in line with the expectations of the thermodynamic model

A key testable prediction stemming from the thermodynamic model is that DvH-s (as well as DvH-ns 1 and 2) clones should produce more hydrogen under sulfate-limited conditions. We verified this prediction by inoculating all 10 sequenced clones on a lactate minimal medium without sulfate, and with $50 \%$ and $100 \%$ of the sulfate needed to degrade all available lactate via the sulfate pathway (see Materials and methods). We found that all DvH-s clones, as well as DvH-ns 1 and 2 clones, produce significant hydrogen above background levels in the absence of sulfate or at $50 \%$ sulfate $(P \leqslant 0.01$, two-tailed $t$-test), while DvH-ns 3, 4 and 5 clones only produce hydrogen significantly above background levels at $50 \%$ sulfate $(P \leqslant 0.05$, two-tailed $t$-test) (Figure 5$)$. The cumulative hydrogen pressure achieved by the former clones over the course of 7 days, in the absence of sulfate and at stationary state is $\sim 300 \mathrm{~Pa}$, well above the pressure needed to sustain $\mathrm{Mm}$ growth. This explains the ability of the DvH-s clones to engage in syntrophy with $\mathrm{Mm}$.

A second prediction stemming from the thermodynamic model discussed above is that hydrogen production of syntrophic DvH-s clones should be affected by modifications in the ion content of the media. The observed cumulative hydrogen pressure of $\sim 300 \mathrm{~Pa}$ from DvH-s clones shows that indeed there is an energy investment into the lactate oxidation within this genotype. Dependent on physiological equilibrium concentrations of lactate, pyruvate and the magnitude of the ion gradient across the membrane, this investment could be achieved by Coo translocating as low as $1 \mathrm{H}^{+} / \mathrm{Na}^{+}$per hydrogen produced (in Figure 4 we show that this investment needs to be $\sim-23 \mathrm{~kJ} \mathrm{~mol}^{-1}$, which under standard conditions equates to translating 2 single charged ions over a 100-fold ion gradient per hydrogen produced). At any rate, changing the $\mathrm{Na}^{+}$concentration in the media should affect the membrane ion gradient, and thereby the level of energy investment and the steady-state hydrogen pressure. To test this, we transferred triplicates of DvH-s and DvH-ns cultures into $\mathrm{Na}$-buffer at different $\mathrm{Na}^{+}$concentrations, and after 6 days we measured the equilibrium hydrogen pressure. Although these cultures did not show any detectable growth, the DvH-s clones produced consistently more hydrogen than the DvH-ns clones, and such production increased with increasing $\mathrm{Na}^{+}$concentration up to a threshold high concentration (Figure 6). The low hydrogen
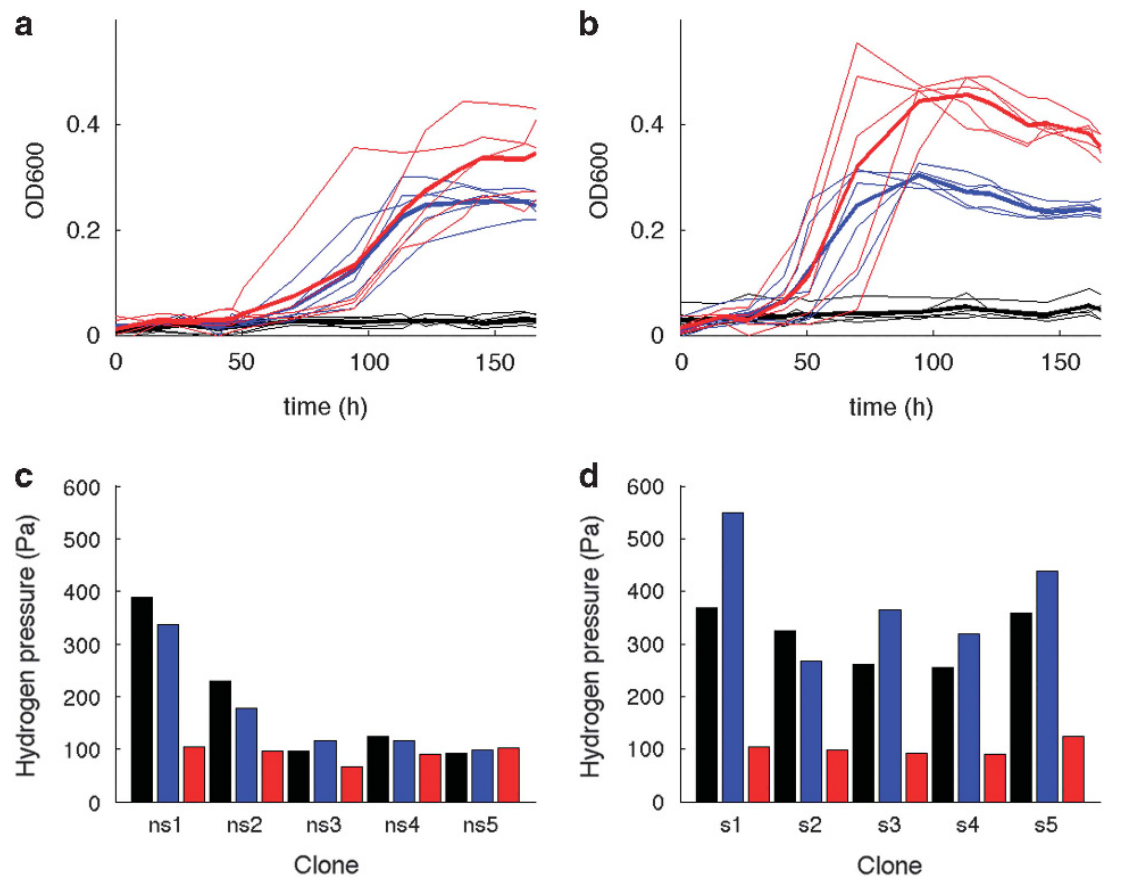

Figure 5 OD600 (a and b, thick lines are mean of five thin lines) and cumulative hydrogen pressure (c and d) after $168 \mathrm{~h}$ of incubation for isolated DvH-ns (a and $\mathbf{c}$ ) and DvH-s (b and $\mathbf{d}$ ) clones. Cultures were grown on CCM containing 30 mm lactate and varying amounts of $\mathrm{SO}_{4}^{2-}$. Black: $0 \mathrm{~mm} \mathrm{SO}_{4}^{2-}$, blue: $7.5 \mathrm{~mm} \mathrm{SO}_{4}^{2-}$, red: $15 \mathrm{~mm} \mathrm{SO}_{4}^{2-}$ (see Materials and methods). Background (ambient) hydrogen pressure was $90 \mathrm{~Pa}$. 


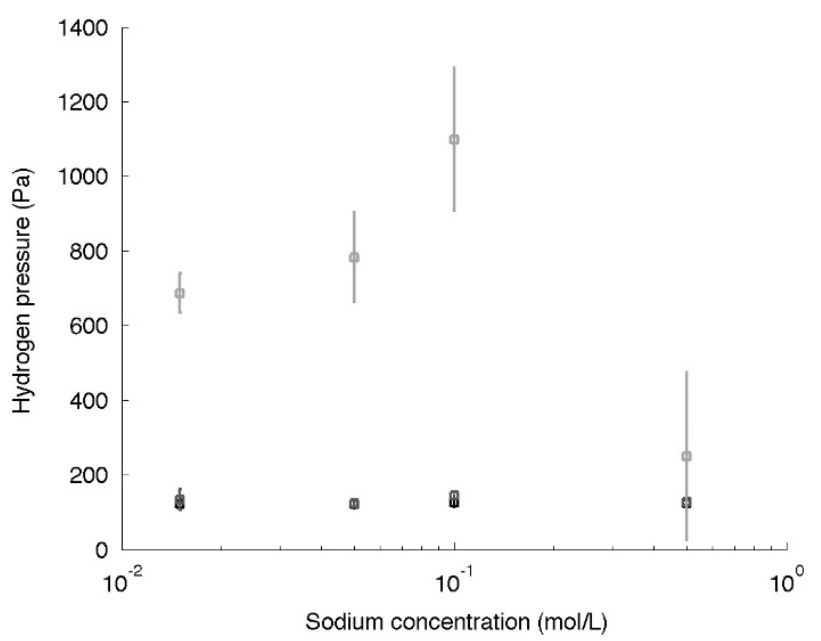

Figure 6 Mean hydrogen pressure and standard deviation of triplicate measurements of DvH-s (red), DvH-ns (blue) and blank medium (black) at different concentrations of sodium. Headspace measurements were performed after 6 days of incubation without detectable growth in the Na-Buffer (see Materials and methods). A full color version of this figure is available at the The ISMEJ Journal journal online.

production at $500 \mathrm{~mm} \mathrm{Na}^{+}$indicates that at that level of $\mathrm{Na}^{+}$concentration the salinity tolerance of DvH is exceeded (Zhou et al., 2013).

\section{Conclusions}

It is well known that SRBs can act as both hydrogen consumers and producers, taking the latter role in the absence of terminal electron acceptors such as sulfate (Muyzer and Stams, 2008). This is believed to underpin the ability of SRBs to occupy different niches and survive different environmental conditions (Muyzer and Stams, 2008). Our findings suggest that the dual physiology is encoded in $\mathrm{DvH}$ as two separate genotypes, which are maintained at different frequencies. As indicated by theoretical studies of phenotypic variability under fluctuating environments (Kussell and Leibler, 2005), it is possible that these $\mathrm{DvH}$ genotype frequencies are in alignment with the frequency of the fluctuations in the environment in terms of availability of strong electron acceptors such as sulfate. At the same time, it is also clear that the frequency at which the syntrophic DvH genotype is maintained will be directly linked to the presence of syntrophic partners such as $\mathrm{Mm}$ in the environment. It remains to be seen if a stable polymorphism as seen in DvH is a strategy common to other SRBs in the environment. From a more applied point, this study points to engineering of thermodynamic constraints in metabolism as a possible route to the implementation of syntrophic interactions in synthetic microbial communities.

\section{Conflict of Interest}

The authors declare no conflict of interest.

\section{Acknowledgements}

We acknowledge members of The Genome Analysis Centre (TGAC) Platforms and Pipelines Group (P\&P) for their help in library construction and sequencing. We thank Angus Buckling for comments on an earlier version of this manuscript and John Leigh and James Chong for their help to establish growth protocols for Mm. We thank three anonymous reviewers for helping to improve the manuscript. This work is funded by a research grant from Biotechnological and Biological Research Council (BBSRC) of the UK (grant ID: BB/K003240/2).

\section{References}

Arnér ESJ. (2010). Selenoproteins-What unique properties can arise with selenocysteine in place of cysteine? Exp Cell Res 316: 1296-1303.

Balaban NQ, Merrin J, Chait R, Kowalik L, Leibler S. (2004). Bacterial persistence as a phenotypic switch. Science 305: 1622-1625.

Bennett BD, Kimball EH, Gao M, Osterhout R, Van Dien SJ, Rabinowitz JD. (2009). Absolute metabolite concentrations and implied enzyme active site occupancy in Escherichia coli. Nat Chem Biol 5: 593-599.

Bernstein HC, Paulson SD, Carlson RP. (2012). Synthetic Escherichia coli consortia engineered for syntrophy demonstrate enhanced biomass productivity. I Biotechnol 157: 159-166.

Cingolani P, Platts A, Wang LL, Coon M, Nguyen T, Wang L et al. (2012). A program for annotating and predicting the effects of single nucleotide polymorphisms, SnpEff: SNPs in the genome of Drosophila melanogaster strain w1118; iso-2; iso-3. Fly (Austin) 6: 1-13.

Cord-Ruwisch R, Seitz H-J, Conrad R. (1988). The capacity of hydrogentrophic anaerobic bacteria to compete for traces of hydrogen depends on the redox potential of the electron acceptor. Arch Microbiol 149: $350-357$.

Doddema HJ, Vogels GD. (1978). Improved identification of methanogenic bacteria by fluorescence microscopy. Appl Environ Microbiol 36: 752-754.

Gilbert ES, Walker AW, Keasling JD. (2003). A constructed microbial consortium for biodegradation of the organophosphorus insecticide parathion. Appl Microbiol Biotechnol 61: 77-81.

Großkopf T, Soyer OS. (2014). Synthetic microbial communities. Curr Opin Microbiol 18: 72-77.

Großkopf T, Soyer OS. (2016). Microbial diversity arising from thermodynamic constraints. ISME $J$ 10: 2725-2733.

Gutiérrez-Sánchez C, Rüdiger O, Fernández V, De Lacey A, Marques M, Pereira I. (2010). Interaction of the active site of the $\mathrm{Ni}-\mathrm{Fe}-\mathrm{Se}$ hydrogenase from Desulfovibrio vulgaris Hildenborough with carbon monoxide and oxygen inhibitors. J Biol Inorg Chem 15: 1285-1292.

Hastings J, de Matos P, Dekker A, Ennis M, Harsha B, Kale N et al. (2013). The ChEBI reference database and 
ontology for biologically relevant chemistry: enhancements for 2013. Nucleic Acids Res 41: D456-D463.

Heidelberg JF, Seshadri R, Haveman SA, Hemme CL, Paulsen IT, Kolonay JF et al. (2004). The genome sequence of the anaerobic, sulfate-reducing bacterium Desulfovibrio vulgaris Hildenborough. Nat Biotechnol 22: 554-559.

Hillesland KL, Stahl DA. (2010). Rapid evolution of stability and productivity at the origin of a microbial mutualism. Proc Natl Acad Sci USA 107: 2124-2129.

Joshi N, Fass J. (2011), Sickle: A sliding-window, adaptive, quality-based trimming tool for FastQ files (Version 1.33) [Software]. Available at https://github.com/ najoshi/sickle, 2011.

Kato S, Watanabe K. (2010). Ecological and evolutionary interactions in syntrophic methanogenic consortia. Microbes Environ 25: 145-151.

Kerner A, Park J, Williams A, Lin XN. (2012). A programmable Escherichia coli consortium via tunable symbiosis. PLoS One 7: e34032.

Kleerebezem R, Stams AJM. (2000). Kinetics of syntrophic cultures: a theoretical treatise on butyrate fermentation. Biotechnol Bioeng 67: 529-543.

Koboldt DC, Zhang Q, Larson DE, Shen D, McLellan MD, Lin L et al. (2012). VarScan 2: somatic mutation and copy number alteration discovery in cancer by exome sequencing. Genome Res 22: 568-576.

Kussell E, Leibler S. (2005). Phenotypic diversity, population growth, and information in fluctuating environments. Science 309: 2075-2078.

Li H, Durbin R. (2010). Fast and accurate long-read alignment with Burrows-Wheeler transform. Bioinformatics 26: 589-595.

Li H, Handsaker B, Wysoker A, Fennell T, Ruan J, Homer N et al. (2009). The Sequence Alignment/Map format and SAMtools. Bioinformatics 25: 2078-2079.

Markowitz VM, Chen I-MA, Palaniappan K, Chu K, Szeto E, Grechkin Y et al. (2012). IMG: the integrated microbial genomes database and comparative analysis system. Nucleic Acids Res 40: D115-D122.

Marx CJ. (2009). Microbiology. Getting in touch with your friends. Science 324: 1150-1151.

McInerney M, Sieber J, Gunsalus R. (2009). Syntrophy in anaerobic global carbon cycles. Curr Opin Biotechnol 20: $623-632$.

McInerney M, Struchtemeyer C, Sieber J, Mouttaki H, Stams A, Schink B et al. (2008). Physiology, ecology, phylogeny and genomics of microorganisms capable of syntrophic metabolism. Ann NY Acad Sci 1125: $58-72$.

Mee MT, Collins JJ, Church GM, Wang HH. (2014). Syntrophic exchange in synthetic microbial communities. Proc Natl Acad Sci USA 111: E2149-E2156.

Morgat A, Axelsen KB, Lombardot T, Alc R, Aimo L, Zerara $\mathrm{M}$ et al. (2015). Updates in Rhea-a manually curated resource of biochemical reactions. Nucleic Acids Res 43: D459-D464.

Morris BEL, Henneberger R, Huber H, Moissl-Eichinger C. (2013). Microbial syntrophy: interaction for the common good. FEMS Microbiol Rev 37: 384-406.

Muyzer G, Stams AJM. (2008). The ecology and biotechnology of sulphate-reducing bacteria. Nat Rev Microbiol 6: 441-454.

Nadell CD, Xavier JB, Foster KR. (2009). The sociobiology of biofilms. FEMS Microbiol Rev 33: 206-224.
Noguera D, Brusseau G, Rittmann B, Stahl D. (1998). A unified model describing the role of hydrogen in the growth of desulfovibrio vulgaris under different environmental conditions. Biotechnol Bioeng 59: 732-746.

Pieulle L, Morelli X, Gallice P, Lojou E, Barbier P, Czjzek M et al. (2005). The type I/type II cytochrome c3 complex: An electron transfer link in the hydrogen-sulfate reduction pathway. J Mol Biol 354: 73-90.

Postgate JR. (1984). The Sulfate Reducing Bacteria, 2nd edn. Cambridge University Press: Cambridge.

Postgate JR, Kent HM, Robson RL, Chesshyre JA. (1984). The genomes Desulfovibrio gigas and D. vulgaris. J Gen Microbiol 130: 1597-1601.

Qi Z, Pei G, Chen L, Zhang W. (2014). Single-cell analysis reveals gene-expression heterogeneity in syntrophic dual-culture of Desulfovibrio vulgaris with Methanosarcina barkeri. Sci Rep 4: 7478.

Santala S, Karp M, Santala V. (2014). Rationally engineered synthetic coculture for improved biomass and product formation. PLoS One 9: e113786.

Schink B. (1997). Energetics of syntrophic cooperation in methanogenic degradation. Microbiol Mol Biol Rev 61: 262-280.

Seitz H, Schink B, Conrad R. (1988). Thermodynamics of hydrogen metabolism in methanogenic cocultures degrading ethanol or lactate. FEMS Microbiol Lett 55: 119-124.

Shimoyama T, Kato S, Ishii S, Watanabe K. (2009). Flagellum mediates symbiosis. Science 323: 1574.

Shou W, Ram S, Vilar JMG. (2007). Synthetic cooperation in engineered yeast populations. Proc Natl Acad Sci USA 104: 1877-1882.

Sieber JR, McInerney MJ, Gunsalus RP. (2012). Genomic insights into syntrophy: the paradigm for anaerobic metabolic cooperation. Annu Rev Microbiol 66: 429-452.

Stolyar S, Van Dien S, Hillesland KL, Pinel N, Lie TJ, Leigh JA et al. (2007). Metabolic modeling of a mutualistic microbial community. Mol Syst Biol 3: 92.

Summers ZM, Fogarty HE, Leang C, Franks AE, Malvankar NS, Lovley DR. (2010). Direct exchange of electrons within aggregates of an evolved syntrophic coculture of anaerobic bacteria. Science 330: 1413-1415.

Thauer RK, Jungermann K, Decker K. (1977). Energy conservation in chemotrophic anaerobic bacteria. Bacteriol Rev 41: 100-180.

Valente FM, Oliveira AS, Gnadt N, Pacheco I, Coelho AV, Xavier AV et al. (2005). Hydrogenases in Desulfovibrio vulgaris Hildenborough: Structural and physiologic characterisation of the membrane-bound [NiFeSe] hydrogenase. J Biol Inorg Chem 10: 667-682.

Walker CB, He Z, Yang ZK, Ringbauer JA, He Q, Zhou J et al. (2009). The electron transfer system of syntrophically grown Desulfovibrio vulgaris. J Bacteriol 191: 5793-5801.

Walker CB, Redding-Johanson AM, Baidoo EE, Rajeev L, He Z, Hendrickson EL et al. (2012). Functional responses of methanogenic archaea to syntrophic growth. ISME J 6: 2045-2055.

Wintermute EH, Silver P a. (2010a). Dynamics in the mixed microbial concourse. Genes Dev 24: 2603-2614.

Wintermute EH, Silver P a. (2010b). Emergent cooperation in microbial metabolism. Mol Syst Biol 6: 407.

Worm P, Koehorst JJ, Visser M, Sedano-Núñez VT, Schaap PJ, Plugge CM et al. (2014). A genomic view on syntrophic versus non-syntrophic lifestyle in anaerobic fatty acid 
degrading communities. Biochim Biophys Acta 1837: 2004-2016.

Zhang W, Culley DE, Nie L, Scholten JCM. (2007). Comparative transcriptome analysis of Desulfovibrio vulgaris grown in planktonic culture and mature biofilm on a steel surface. Appl Microbiol Biotechnol 76: 447-457.

Zhou A, Baidoo E, He Z, Mukhopadhyay A, Baumohl JK, Benke P et al. (2013). Characterization of NaCl tolerance in Desulfovibrio vulgaris Hildenborough through experimental evolution. ISME J 7: 1790-1802.
This work is licensed under a Creative Commons Attribution 4.0 International License. The images or other third party material in this article are included in the article's Creative Commons license, unless indicated otherwise in the credit line; if the material is not included under the Creative Commons license, users will need to obtain permission from the license holder to reproduce the material. To view a copy of this license, visit http:// creativecommons.org/licenses/by/4.0/

Supplementary Information accompanies this paper on The ISME Journal website (http://www.nature.com/ismej) 